RELACIÓN DEL FACTOR INDIVIDUAL CON LA FUNCIÓN INVESTIGACIÓN EN LAS UNIVERSIDADES PÚBLICAS.

\title{
RELACIÓN DEL FACTOR INDIVIDUAL CON LA FUNCIÓN INVESTIGACIÓN EN LAS UNIVERSIDADES PÚBLICAS
}

\section{THE RELATION BETWEEN THE INDIVIDUAL FACTOR AND THE RESEARCH FUNCTION IN PUBLIC UNIVERSITIES}

Deisy María Jerónimo Jiménez*, Abel Pérez Sánchez**, Salvador Neme Calacich***

*Maestra en Administración. Candidata a Doctor en Administración por la Universidad Autónoma de Guadalajara, Campus Tabasco. ORCID: https://orcid.org/0000-0003-2922-3251.

**Doctor en Administración. Profesor de la Universidad Autónoma de Guadalajara, Campus Tabasco. Email: aperezs@uagtabasco.edu.mx. ORCID: https://orcid.org/0000-0002-0088-1122.

***Doctor en Gestión Estratégica y Políticas de Desarrollo. Profesor Investigador de la Universidad Juárez Autónoma de Tabasco. Email: s_neme@hotmail.com. ORCID: https://orcid.org/0000-0003-4085-9541.

Dirección para correspondencia: deimamx@yahoo.com.mx 
RELACIÓN DEL FACTOR INDIVIDUAL CON LA FUNCIÓN INVESTIGACIÓN EN LAS UNIVERSIDADES PÚBLICAS.

\section{RESUMEN}

OBJETIVO: Conocer la relación de la variable factor individual del aprendizaje organizacional con el desempeño en la productividad científica del personal académico de una Institución de Educación Superior Pública ubicada en el sureste mexicano.

MATERIAL Y MÉTODO: Se aplicó un instrumento basado en la teoría del aprendizaje organizacional y específicamente del factor individual, mismo que se conformó por dos variables: altruismo y autoeficacia.

RESULTADOS: Fue un instrumento integrado por 31 ítems, el cual obtuvo .91 de Alfa de Cronbach, logrando encuestar a 190 profesores de tres divisiones académicas del área de conocimientos de ciencias sociales. Para analizar y explicar la variable dependiente "productividad científica" se realizó la técnica estadística de regresión lineal múltiple, utilizando como variables independientes las sociodemográficas y "factor individual", obteniendo como resultado que el factor individual es el que más aporta a la variable dependiente, no siendo así la edad, la cual no se considera estadísticamente significativa.

CONCLUSIONES: Se puede observar, que el factor individual con el $50.4 \%$ es el que más aporta a la productividad científica, donde el altruismo y la autoeficacia percibida por el docente son de importancia, con esto, se logra demostrar la relación del factor individual del aprendizaje organizacional para la investigación como mediador en la productividad científica.

PALABRAS CLAVE: Investigación. Instituciones de Educación Superior. Aprendizaje organizacional.

\section{ABSTRACT}

OBJECTIVE: To know the relation between the variable individual factor in organizational learning with performance in faculty scientific productivity from a Public Higher Education Institution located in the southeast Mexico. 
RELACIÓN DEL FACTOR INDIVIDUAL CON LA FUNCIÓN INVESTIGACIÓN EN LAS UNIVERSIDADES PÚBLICAS.

MATERIAL AND METHOD: An instrument based on organizational learning theory, specifically on the individual factor, was administered, formed by two variables: altruism and selfeffectiveness.

RESULTS: The instrument was integrated by 31 items, which obtained a .91 Cronbach Alfa, administered to 190 professors from three academic divisions from Social Sciences. In order to analize and explain the dependent variable "scientific productivity", a multiple linear statistic regression technique was used, as independent variables individual factor and sociodemographics were considered, obtaining as a result that the individual factor has an impact on the dependent variable, not age, and it is not statistically significant.

CONCLUSIONS: It can be observed that the individual factor with $50.4 \%$ has an impact on scientific productivity where altruism and self-effectiveness are perceived and considered of importance by professors, then proving the relation between the individual factor of organizational learning towards research as a mediator in the scientific productivity.

PALABRAS CLAVE: Research. Higher Education Institutions. Organizational Learning.

\section{INTRODUCCIÓN}

En un mundo globalizado el entorno es inestable para las organizaciones; estas compiten en mercados abiertos, tanto nacional como internacional. Los recursos financieros y recursos tecnológicos tienden a estar al alcance de todos en igualdad de condiciones, así que lo que hace la diferencia es el conocimiento; lo anterior, causó que el recurso humano se viera de otra manera, al ser ellos quienes tienen el conocimiento. (Pérez, Montes y Vázquez, 2006).

En la década de los noventa se inician estudios del cómo hacer que el conocimiento se produzca y transfiera en la organización; lo que marcaría los inicios de las teorías: "gestión del conocimiento" (Nonaka, Byosiere, Borucki y Konnot, 1994), "aprendizaje organizacional" (Argyris, 1991; Huber 1991) y "capital intelectual" (Bontis, 1998). "En todas ellas el conocimiento generado es un medio para lograr los objetivos planteados por la organización y un activo intangible" (Davenport, De Long y Beers, 1998), donde el aprendizaje organizacional permite que "el conocimiento no quede estancado, sino que sea un proceso dinámico al ser actualizado 
RELACIÓN DEL FACTOR INDIVIDUAL CON LA FUNCIÓN INVESTIGACIÓN EN LAS UNIVERSIDADES PÚBLICAS.

y compartido por sus miembros dentro de la organización" (Alshehhi y Jasimuddin, 2016; Bontis, Crossan, y Hulland, 2002), "generando conocimiento y habilidades en los empleados, que la organización se apropia para lograr desarrollo y crecimiento organizacional" (Pérez, et al., 2006; Saadat y Saadat, 2016), "utilizado como una herramienta estratégica para obtener ventaja competitiva y estabilizar el éxito de la organización" (Lee y Choi, 2003; Lin, 2007; Pérez, et al., 2006).

"Una forma de evidenciar la efectividad del aprendizaje organizacional, es con el desempeño organizacional, para lo cual el recurso humano, estructura organizacional y tecnología son necesarios" (Lin, 2007; Ordónez de Pablos y Lytras, 2008; Pérez, et al., 2006). "El desempeño ha sido medido con la productividad, estructura y capital humano" (Parra y Calderón, 2013).

"De estas tres variables relacionadas al desempeño organizacional, la productividad es el resultado de la contribución del capital humano" (Del Castillo, y Vargas, 2009), por lo cual "de acuerdo a la contribución que hace a la organización se le valora" (Pedraza, Amaya, y Conde, 2010). "Bajo este enfoque, el recurso humano ha adquirido mayor relevancia, porque es un recurso de valor incalculable, este posee los conocimientos y habilidades para producir" (Almanza, Calderón, Vargas, Casas, y Palomares, 2016; Saadat y Saadat, 2016; y Toledo, 2009), "además que sin ellos la organización no puede existir" (Pérez, et al., 2006).

"En este escenario el papel de la gestión de recursos humanos se ha replanteado por varios investigadores. Originalmente consistía en reclutamiento, evaluación y recompensas, ahora se ha añadido la variable aprendizaje" (Abdullah y Elgaraihy, 2013; Pérez, et al., 2006; Seleim, Ashour, y Bontis, 2007). Considerando lo anterior, una de las discusiones teóricas de la gestión del recurso humano "es lo que permite que los empleados sean más productivos" (Drucker, 2000).

Por lo tanto, este estudio de investigación se basa en las variables que regulan las actividades de los empleados y que influyen en su desempeño o productividad; de acuerdo a Porter y Wayland (1992) "el desarrollo de capacidades, formación de los empleados, sistemas de información, desarrollo organizacional y vinculación con el exterior están interrelacionados como partes fundamentales de la productividad". 
RELACIÓN DEL FACTOR INDIVIDUAL CON LA FUNCIÓN INVESTIGACIÓN EN LAS UNIVERSIDADES PÚBLICAS.

Una interrogante es, si la productividad en el empleado se debe al aprendizaje a través de la especialización con mecanismos formales de la organización, como es la capacitación o si hay otras formas para lograr el aprendizaje que a simple vista no se distinguen, considerando que los empleados son parte de un entorno laboral y social (Toledo, 2009; Yanez, Hernandez y Moreno, 2017), lo que les ha permitido aprender de otros; porque la capacidad de aprendizaje de los seres humanos está en función de aspectos individuales y sociales (Toledo, 2009).

Silva y Flores (2015) añadieron la necesidad de considerar las capacidades y habilidades de los empleados para realizar sus actividades de manera correcta. Al respecto, Lin (2007) agrega que "los empleados pueden estar motivados por el altruismo el deseo de ayudar a otros, lo cual está relacionado con la autoeficacia; es decir, tener la confianza en que saben".

"En estos estudios, la estructura organizacional ha sido señalada como parte de las acciones dirigidas a los procesos de aprendizaje en los individuos, grupos y la propia organización" (Cortes y Pérez, 2008; Lin, 2007); “integrada por las normas, reglas y procesos, que permite al capital humano conocer las condiciones laborales, actividades a realizar, tiempo para efectuarlas, responsabilidades, exigencias, incentivos y los recursos o apoyos necesarios que aseguran su desempeño-resultado" (Ruiz, Silva y Vanga, 2008). Según Lin y Lee (2006) "la flexibilidad o inflexibilidad en la formalidad de políticas y reglamentos dentro de la organización han facilitado o impedido este tipo de aprendizaje, por lo cual recomiendan la flexibilidad para el éxito".

En el contexto donde mayormente se ha investigado el aprendizaje organizacional ha sido en las empresas, y se han rezagado las instituciones públicas, principalmente las educativas, generadoras de conocimiento, para la cual Lin (2007); y Zakaria y Taiwo (2013) recomendaron "para futuras investigaciones examinar el tamaño de la empresa y el tipo de industria, porque pueden moderar las relaciones de los factores del aprendizaje".

Bajo esta premisa, este trabajo se centra en una Institución de Educación Superior (IES), debido a que son vistas como organizaciones que generan conocimiento a través de investigaciones que realizan su personal académico, vinculándose con empresas, para que estas últimas innoven en el desarrollo de procesos tecnológicos y creen nuevos productos o servicios y logren ser líderes en el mundo actual (Awadh, 2007; Cárdenas, Cabrero y Arellano, 
RELACIÓN DEL FACTOR INDIVIDUAL CON LA FUNCIÓN INVESTIGACIÓN EN LAS UNIVERSIDADES PÚBLICAS.

2012; Consejo Nacional de Ciencia y Tecnología [CONACYT], 2008, Organización de las Naciones Unidas para la Educación, la Ciencia y la Cultura [UNESCO], 1998).

"Una forma de medir la productividad en la investigación es con la cantidad de trabajos publicados en un periodo de tiempo" (Hadjinicola y Soteriou, 2006; y Vásquez, 2010). Stephan (1996 citado por Mollick, 2012) en su trabajo de investigación encontró que, "en este tipo de productividad, sólo el $6 \%$ de los investigadores es responsable del $50 \%$ de todas las publicaciones, concluyendo que la diferencia se debe en parte a distintas capacidades entre ellos".

En México, en el periodo 2016-2017, la participación porcentual fue de 0.66\% en la producción total mundial de artículos de entre 35 naciones integrantes de los países miembros de la Organización para la Cooperación y el Desarrollo Económicos (OCDE), ubicándose en el lugar 19, lista encabezada por Estados Unidos con 16.92\%; de 10 áreas temáticas, la que presenta el mayor volumen de artículos en plantas y animales con un 12.61\%; mientras que el área de Ciencias Sociales 4.51\%, ubicándose en el último lugar; además el 99\% de las IES públicas mexicanas a nivel mundial no tienen presencia, ni reconocimiento en la productividad científica (CONACYT, 2017).

El Programa para el Desarrollo Profesional Docente (PRODEP), surge como una forma de motivar a los profesores de tiempo completo (PTC) de las IES públicas, para que desarrollen aptitudes de investigación además de la docencia; dicho programa los evalúa y si cumplen con la productividad científica, obtienen el reconocimiento perfil deseable; sin embargo, de las 731 IES sólo 536 están en este programa (ver tabla 1), donde hay una población de 55,539 PTC con posgrado en el año 2018, y 28,550 lograron el reconocimiento mencionado, lo que representa solo el $51.41 \%$ (ver tabla 2 ).

También el padrón de beneficiarios del Sistema Nacional de Investigadores reportó 28,634 investigadores reconocidos por este sistema (SNI, CONACYT, 2018); de este padrón solo en 15 IES públicas se concentran 14,038 y cuatro de estas 15 IES centralizan el $50.76 \%$ las cuáles son del Subsistema de Instituciones Federales, y el resto en 11 Universidades Públicas Estatales. En conclusión, de los 55,539 PTC con posgrado, de acuerdo al SNI solo el 25.27\% 
RELACIÓN DEL FACTOR INDIVIDUAL CON LA FUNCIÓN INVESTIGACIÓN EN LAS UNIVERSIDADES PÚBLICAS.

de profesores en IES realiza investigaciones, genera conocimiento y tiene productividad científica.

\section{Tabla 1}

Número de instituciones por subsistema en educación superior y beneficiadas por PRODEP ${ }^{1}$

\begin{tabular}{|c|c|c|c|}
\hline Subsistema & Siglas & Número & $\begin{array}{l}\text { Beneficiadas } \\
\text { por PRODEP }\end{array}$ \\
\hline Universidades Públicas Estatales & UPE & 34 & 34 \\
\hline $\begin{array}{l}\text { Universidades Públicas Estatales de Apoyo } \\
\text { Solidario }\end{array}$ & UPEA & 23 & 20 \\
\hline Instituciones Federales & IF & 8 & 7 \\
\hline Universidades Tecnológicas & UT & 107 & 92 \\
\hline Universidades Politécnicas & UPT & 51 & 51 \\
\hline Institutos Tecnológicos Federales & IT & 132 & 99 \\
\hline Institutos Tecnológicos Descentralizados & ITD & 103 & 100 \\
\hline Escuelas Normales & EN & 260 & 123 \\
\hline Universidades Interculturales & UIC & 13 & 10 \\
\hline Total & & 731 & 536 \\
\hline
\end{tabular}

http://www.dgesu.ses.sep.gob.mx/PRODEP.htm, del cuarto trimestre 2018.

\footnotetext{
${ }^{1}$ Programa para el Desarrollo Profesional Docente, para el Tipo Superior (PRODEP). IES atendidas en el 4to. Trimestre de 2018.
} 
RELACIÓN DEL FACTOR INDIVIDUAL CON LA FUNCIÓN INVESTIGACIÓN EN LAS UNIVERSIDADES PÚBLICAS.

Tabla 2

Total de PTC por subsistema con reconocimiento Perfil Deseable PRODEP 2

\begin{tabular}{|c|c|c|c|c|}
\hline Institución & $\begin{array}{l}\text { Total de } \\
\text { PTC }\end{array}$ & $\begin{array}{l}\text { PTC con } \\
\text { posgrado }\end{array}$ & $\begin{array}{l}\text { Con Perfil Deseable } \\
\text { Vigente }\end{array}$ & $\% *$ \\
\hline Universidades Públicas Estatales & 33,681 & 32,010 & 20,181 & $63.05 \%$ \\
\hline $\begin{array}{l}\text { Universidades Públicas Estatales de } \\
\text { Apoyo Solidario }\end{array}$ & 2,193 & 1,889 & 812 & $42.99 \%$ \\
\hline Instituciones Federales & 5,991 & 4,863 & 1,951 & $40.12 \%$ \\
\hline Universidades Tecnológicas & 4,801 & 2,965 & 1,397 & $47.12 \%$ \\
\hline Universidades Politécnicas & 1,680 & 1,623 & 691 & $42.58 \%$ \\
\hline Institutos Tecnológicos & 10,936 & 5,908 & 1,754 & $29.69 \%$ \\
\hline Institutos Tecnológicos Descentralizados & 4,045 & 2,650 & 1,052 & $39.70 \%$ \\
\hline Escuelas Normales & 5,358 & 3,330 & 601 & $18.05 \%$ \\
\hline Universidades Interculturales & & 301 & 111 & $36.88 \%$ \\
\hline Total & 69,070 & 55,539 & 28,550 & $51.41 \%$ \\
\hline
\end{tabular}

http://www.dgesu.ses.sep.gob.mx/PRODEP.htm, del cuarto trimestre 2018.

*Porcentaje de Perfil deseable obtenido sobre los PTC con posgrado.

"Los seres humanos nacen con una capacidad innata para aprender, a través de la cual se adaptan a los cambios y desafíos ambientales" (Bavarsad, Rahimi, Beiranvand, y Jafarnea, 2016), "con el aprendizaje adquieren conocimientos técnicos y habilidades, desarrollan la capacidad física necesaria y producen algún tipo de acción, también adquieren conocimientos para desarrollar la capacidad de comprensión conceptual sobre una experiencia; es decir, por qué" (Kim, 1993).

"Desde la perspectiva de gestión para la competitividad organizacional, el aprendizaje organizacional es el proceso dinámico de creación, adquisición e integración del conocimiento

\footnotetext{
2 Programa para el Desarrollo Profesional Docente, para el Tipo Superior (PRODEP). IES atendidas en el 4to. Trimestre de 2018.
} 
RELACIÓN DEL FACTOR INDIVIDUAL CON LA FUNCIÓN INVESTIGACIÓN EN LAS UNIVERSIDADES PÚBLICAS.

dirigido a desarrollar los recursos y capacidades que permiten a la organización lograr un mejor rendimiento" (Jyoti, Gupta, y Kotwal, 2011; Lee y Choi, 2003; Lin, 2007; Pérez, et al., 2006), "incrementando el conocimiento a través de buenas prácticas del aprendizaje individual, grupal y organizacional" (Broadbent, 1998).

"En estudios anteriores, el aprendizaje se ha medido con las dimensiones factor individual, factor estructura organizacional y factor tecnológico" (Lin, 2007) "y se ha demostrado que el aprendizaje organizacional es un moderador para lograr el desempeño" (Lee y Choi, 2003, Zack, McKeen, y Singh, 2009).

En este trabajo se analiza el factor individual "pues el aprendizaje organizacional inicia con ellos" (Apórtela y Ponjuán, 2008); es decir, se refiere a las cualidades que tiene el ser humano para adquirir y compartir conocimiento y se deben considerar características individuales, personalidad, competencias relacionadas a las aptitudes y habilidades, creencias y motivaciones, las cuales se reflejan en el comportamiento de la persona (Chiavenato, 2009).

Huber (1991), dice que "la experiencia, observación y búsqueda de información sobre el entorno de la organización crean o generan conocimientos en el individuo"; "esta experiencia puede ser directa o a través de otros, alianzas estratégicas o vinculación con los clientes, redes, funciones de integración, reuniones de trabajo, evaluación de la resolución efectiva de conflictos, trabajo en equipo" (Pérez, et al., 2006).

López, Pérez, y Valle (2009) demostraron una relación positiva con la creación del conocimiento y el desarrollo interno del recurso humano, como la capacitación, desarrollo, evaluación y compensación; cuando el individuo se considera autoeficaz, desarrolla una mayor disposición para compartir y recibir conocimiento, pues está seguro de tener la capacidad de realizar sus actividades de manera correcta y lograr un mejor desempeño en ellos y compañeros de grupo (Lin, 2007); es decir, "el empleado tiene la confianza de que con sus conocimientos pueden ayudar a otros" (Luthans, 2003 en Lin, 2007). Demostrado por Pérez, et al. (2006) quienes confirmaron en gerentes generales de empresas españolas la relación estadísticamente significativa entre la capacitación y el proceso de adquisición o generación del conocimiento.

En las organizaciones, para lograr el aprendizaje organizacional, es fundamental el compartir conocimiento, por lo que el altruismo se puede considerar como una actitud positiva del 
RELACIÓN DEL FACTOR INDIVIDUAL CON LA FUNCIÓN INVESTIGACIÓN EN LAS UNIVERSIDADES PÚBLICAS.

trabajador para compartir lo que sabe con los demás miembros de la organización. El altruismo procura el bien ajeno aún a costa del propio, con la preocupación o atención desinteresada por el otro (Guinot, Chiva, y Mallén, 2015).

Del mismo modo, se evidencia con un comportamiento racional-individual, tomando en cuenta la situación o condición de los demás, es decir racionalmente el individuo puede decidir colaborar o no colaborar de acuerdo a si lo que espera es un incentivo extrínseco o sólo la satisfacción de ayudar (Pérez, Rodríguez y Sotelo, 2012).

"Estudios que la han contemplado son los realizados en organizaciones que realizan actividades sociales y el personal que labora en ellas es voluntario, no percibe un sueldo, por lo cual se presume su comportamiento es altruista" (Wilson y Musick, 1997).

Con respecto a la variable desempeño laboral, "esta es considerada como el resultado de los empleados al realizar sus actividades y tareas dentro de la organización, cuya finalidad es alcanzar los objetivos propuestos por esta última y así favorecer al desempeño organizacional" (Del Castillo, y Vargas, 2009; Elenkov, 2002; Griffis, Cooper, Golds, y Closs, 2004); "en sí este último desempeño depende directamente del desempeño de las personas que la integran" (Chiavenato, 2009).

El desempeño organizacional se ha medido a través de tres variables: "productividad, estructura organizacional y capital humano" (Parra y Calderón, 2013). "Las tres dimensiones están relacionadas al desempeño organizacional, el capital humano contribuye con su desempeño laboral a los objetivos de la organización, al permitir o impedir el logro de ellos" (Del Castillo, y Vargas, 2009). Por lo cual "el desempeño o productividad del empleado es valorado de acuerdo a su contribución a la organización" (Pedraza, Amaya, y Conde, 2010). Por lo tanto, el objetivo de este estudio es: demostrar la relación del factor individual del aprendizaje organizacional para la investigación como mediador en la productividad científica.

\section{MATERIAL Y MÉTODO}

Este trabajo de investigación se realizó con el personal académico de la Universidad Juárez Autónoma de Tabasco (UJAT), de tres Divisiones Académicas: División Académica de Ciencias Económico Administrativas, División Académica de Ciencias Sociales y Humanidades y la 
RELACIÓN DEL FACTOR INDIVIDUAL CON LA FUNCIÓN INVESTIGACIÓN EN LAS UNIVERSIDADES PÚBLICAS.

División Académica de Educación y Artes, las cuales integran el área de conocimiento de ciencias sociales y administrativas.

Se empleó el enfoque de estudio cuantitativo, para expresar y predecir a través de la teoría administrativa la relación del factor individual del aprendizaje organizacional con la productividad científica del personal académico, y de esa forma explicar la población de estudio de acuerdo a los resultados encontrados en la muestra (Hernández, Fernández, y Baptista, 2010).

El diseño de la investigación fue no experimental, "porque el objeto de estudio se encuestó en su lugar de trabajo en un solo momento" (Hernández, et al., 2010); es decir, en la división académica donde labora. Este diseño fue considerado, debido a que la permanencia de dicho personal dentro de la división es durante su horario de clases, el cual no es el mismo para todos.

El total de la población en las tres divisiones mencionadas fue de 879 profesores, para determinar el tamaño de muestra se utilizó un nivel de confianza del 95\%, con un grado de error del 5\% y una probabilidad de realizar investigación del 19\%, al ser este último los PTC con reconocimiento de perfil PRODEP, que de acuerdo dicho reconocimiento son los que realizan investigación (UJAT, 2018).

El tamaño de la muestra fue de 187 profesores, esta muestra se encuentra en el rango de estudios anteriores, las cuales han sido entre el rango de 90 y 250 (Ahani, Bahrami, y Rostami, 2012; Hadjinicola y Soteriou, 2006; Pérez, et al., 2006; Vásquez, 2010). Para la contestación de la encuesta se eligieron a los docentes de manera probabilística.

La consistencia interna del instrumento fue validado con el análisis estadístico denominado coeficiente de fiabilidad Alfa de Cronbach, herramienta del software estadístico SPSS versión 24, "donde las escalas para evidenciar que los ítems tienen consistencia interna deben oscilar entre .80 y .90." (Hall, 1963); en el cual, el factor individual obtuvo un valor de .91.

Asimismo, se realizó la validez de contenido a los ítems, para que estuvieran de acuerdo al contexto de estudio; se invitó a cinco profesores que hacen investigación para que lo revisaran, durante este proceso se hicieron algunos ajustes y se eliminaron ambigüedades en la redacción 
RELACIÓN DEL FACTOR INDIVIDUAL CON LA FUNCIÓN INVESTIGACIÓN EN LAS UNIVERSIDADES PÚBLICAS.

de algunos ítems de acuerdo a sus recomendaciones. Algunos autores coinciden en que el instrumento se debe enviar a expertos, para que ellos los evalúen en redacción y se eliminen errores o ambigüedades (Ahani, et al., 2012, Pérez, et al., 2006; Vásquez, 2010).

El resultado fue un cuestionario con 31 preguntas, ocho variables demográficas, 12 ítems para medir el factor individual dimensión del aprendizaje organización y 11 ítems para medir la variable productividad científica. El aprendizaje organizacional, de acuerdo a la evidencia empírica encontrada se ha medido con ítems en la escala de Likert en cinco puntos (Hadjinicola y Soteriou, 2006; Lee y Choi, 2003; Lin, 2007; Lin y Lee, 2006; Pérez, et al. 2006), las alternativas son: "nunca" que equivale a cero, hasta "siempre" con valor de cuatro.

\section{RESULTADOS}

La aplicación de la encuesta duró cuatro meses. Inició a mediados del mes de junio y concluyó en la primera semana de septiembre del año 2018; lo anterior, se debió a que el personal objetivo en los meses de junio a julio tiene un horario discontinuo, pues las clases normales del ciclo escolar han concluido y los profesores solo asisten a cursos de capacitación o reuniones de trabajo, esto provocó que durante esos dos meses la recaudación de los datos fuera lenta en comparación de agosto y septiembre.

Para la aplicación de la encuesta se emplearon dos formas: tradicional y electrónica; en la tradicional se fue a los centros de trabajos y se entregó en formato impreso al personal docente; la otra forma fue en línea, por lo que se envió la dirección electrónica a los correo electrónicos o celulares de los profesores, para la creación de la encuesta en línea se empleó la plataforma electrónica del navegador Google. Con el uso de estas dos opciones se obtuvieron 190 encuestas, tres más de las consideradas en el tamaño de muestra (ver tabla 3).

Con el software SPSS versión 24, se realizó la estadística descriptiva de las variables sociodemográficas, como fueron: categoría, antigüedad, nivel de estudios, edad, reconocimientos obtenidos, entre otros.

Se encontró que 133 profesores tienen categoría de tiempo completo, nueve de medio tiempo y 48 de asignatura hora semana mes; de esos profesores 98 son hombres y 92 mujeres; la antigüedad mínima es de un año y la máxima de 43 años, 165 tienen una antigüedad entre 
RELACIÓN DEL FACTOR INDIVIDUAL CON LA FUNCIÓN INVESTIGACIÓN EN LAS UNIVERSIDADES PÚBLICAS.

cinco y 34 años. En lo referente al nivel de estudios, 86 tienen grado de doctor, 91 maestría y 13 sólo licenciatura. Con respecto a la edad, la mínima fue de 25 años y la máxima de 75 , donde 158 profesores están entre 37 y 62 años.

Tabla 3

Profesores encuestados por división académica

\begin{tabular}{lccc}
\hline & \multicolumn{2}{c}{ Género } & Total \\
\cline { 2 - 3 } Divisiones Académicas & Hombre & Mujer & 81 \\
\hline DACEA & 43 & 38 & 43 \\
DACSyH & 28 & 15 & 66 \\
DAEA & 27 & 39 & 190 \\
Total & 98 & 92 & \\
\hline
\end{tabular}

Fuente: Elaborado en SPSS con los datos obtenidos en las encuestas.

"Los reconocimientos al personal por la realización de una actividad son incentivos para que éste realice un mejor desempeño en sus funciones y aumente su productividad" (Martín Cruz, et al., 2009); para este estudio se consideraron los concedidos en el ámbito de la investigación por organismos nacionales y estatales de prestigio.

EI CONACyT otorga el nombramiento Miembro del Sistema Nacional de Investigadores (SNI), la Secretaría de Educación Pública (SEP) a través del PRODEP concede el Reconcomiendo al Perfil Deseable y el Consejo de Ciencia y Tecnología del Estado de Tabasco (CCyTET) el Sistema Estatal de Investigadores (SEI).

De los 190 profesores, 106 tiene algún reconocimiento: 11 han logrado el SNI, 71 SEI y/o 94 PRODEP, en la tabla 4, se observa el número de profesores que han obtenido de uno hasta tres reconocimientos. 
RELACIÓN DEL FACTOR INDIVIDUAL CON LA FUNCIÓN INVESTIGACIÓN EN LAS UNIVERSIDADES PÚBLICAS.

Tabla 4

Clasificación de reconocimientos obtenidos

\begin{tabular}{lccrr}
\hline Reconocimientos & DACEA & DACSyH & DAEA & Total \\
\hline Ninguno & 29 & 28 & 27 & 84 \\
PRODEP & 14 & 8 & 12 & 34 \\
PRODEP,SNI & 0 & 0 & 1 & 1 \\
SEI & 3 & 2 & 7 & 12 \\
SEI, PRODEP & 31 & 3 & 15 & 49 \\
SEI, PRODEP, SNI & 4 & 2 & 4 & 10 \\
Total & 81 & 43 & 66 & 190 \\
\hline
\end{tabular}

Fuente: Elaborado en SPSS con los datos obtenidos en las encuestas.

Asimismo, Se efectuó un análisis de regresión lineal múltiple en el software estadístico SPSS, para tener información estadística y predecir la relación que existe entre las variables independientes sociodemográficas nivel de estudios, antigüedad como profesor, edad y género, así como la variable factor individual del aprendizaje organizacional con la variable dependiente productividad científica, y así obtener la ecuación que muestre esta relación.

En la tabla 5, se observa que la variación de la productividad científica de acuerdo a las respuestas de los profesores encuestados está explicada en un $69.5 \%$ por lo menos por una de las cinco variables independientes. La variable factor individual es la que más la explica al tener un $50.4 \%$, nivel de estudio con un $25.6 \%$, seguida por antigüedad que aporta el $20.4 \%$, género en un $5 \%$, mientras que la variable edad tiene una relación negativa de -10.6\%.

En la columna del análisis de significancia (ANOVA sig.), se observa que entre las variables independientes con la variable dependiente el valor de significancia para $\mathrm{R}$ es de .000 lo cual es menor a .05, al ser menor se establece que existe una relación lineal significativa entre estas variables y la variable dependiente. También en esta tabla se puede observar que la relación lineal con productividad científica es significativa con tres variables, pero no así con las variables género y edad. 
RELACIÓN DEL FACTOR INDIVIDUAL CON LA FUNCIÓN INVESTIGACIÓN EN LAS UNIVERSIDADES PÚBLICAS.

\section{Tabla 5}

Resumen de la relación entre variables sociodemográficas, variable factor individual del aprendizaje organizacional con la variable dependiente productividad científica

\begin{tabular}{lcccc}
\hline Modelo & $\mathrm{R}$ & $\begin{array}{c}\mathrm{R} \\
\text { cuadrado }\end{array}$ & $\begin{array}{c}\text { Coeficientes } \\
\text { estandarizados Beta }\end{array}$ & ANOVA Sig. \\
\hline (Constante) & $.695 \mathrm{a}$ & .483 & & .000 \\
Factor Individual & & .504 & .000 \\
Nivel de estudios & & .256 & .000 \\
Antigüedad (años) & & .204 & .030 \\
Género & & .050 & .364 \\
Edad & & -.106 & .267 \\
\hline
\end{tabular}

Fuente: Elaborado en SPSS con los datos obtenidos en las encuestas.

a. Variable dependiente: Productividad Científica.

\section{CONCLUSIONES}

Al utilizar las variables demográficas nivel de estudios, género y edad, así como la variable factor individual del aprendizaje organizacional, esta última con preguntas acorde al contexto académico en relación a la función sustantiva de la investigación, se analizó la relación que tienen con la productividad científica de los docentes.

En el modelo obtenido al aplicar la regresión lineal múltiple a las variables mencionadas, se puede observar que el factor individual con el $50.4 \%$ es el que más aporta a la productividad científica, donde el altruismo y la autoeficacia percibida por el docente son de importancia en este contexto, con este resultado se logra demostrar la relación del factor individual del aprendizaje organizacional para la investigación como mediador en la productividad científica y cumplir con el objetivo de este estudio.

Lo cual fue congruente con los estudios realizados en el contexto empresarial por Lee y Cho (2003), Lin (2007), Lin y Lee (2006) y Zack, et al. (2009) quienes concluyeron que el aprendizaje organizacional es un moderador para lograr el desempeño en las organizaciones, y 
RELACIÓN DEL FACTOR INDIVIDUAL CON LA FUNCIÓN INVESTIGACIÓN EN LAS UNIVERSIDADES PÚBLICAS.

los empleados deben estar dispuestos para trabajar en equipo, además de tener habilidad técnica de métodos o procedimientos y conocimiento para realizar la actividad.

Cuando el empleado se cree capaz o competente en las tareas que realiza, incrementa su desempeño y control en ella, lo que puede provocar un sentimiento de altruismo y decida compartir sus conocimientos o lo aprendido con sus compañeros en la realización de estas (Lin, 2007; López, et al., 2009; Singh y Chand, 2018;). Resultados que apoyan lo mencionado por Bandura (1978) sobre las expectativas de eficacia del personal, si este se siente autoeficaz hará frente a los obstáculos y persistirá en las actividades que le son subjetivamente amenazantes.

El aporte del $25.6 \%$ que hace el nivel de estudios a la productividad, se puede explicar con lo que señalan Khan, Rajasekar y Al-Asfour (2015) el desarrollo profesional en el factor humano abona a su talento brindándole oportunidades para aprender y crecer en su profesión, incentivándolo a ser efectivo en sus funciones y actividades. En el contexto de organizaciones centradas en el trabajo del conocimiento los aspectos personales como las aptitudes cognitivas explican las diferencias de productividad (Awadh, 2007; Bland, Center, Finstad, Risbey, y Staples, 2006; y Mollick, 2012).

La variable antigüedad aporta un $20.4 \%$, contrario a lo obtenido por Amador, Aguirre, Anguiano, y Guízar (2018) quienes no encontraron relación estadísticamente significativa con la productividad de los empleados del ramo de la industria del calzado. Sin embargo en este contexto su aporte estadísticamente tiene significancia lo cual se puede deber a que en la actividad de la investigación, los años de experiencia permite tener mayor productividad en ella.

La variable género solo aportó un $5 \%$ a la productividad, al respeto Salas y Alegre (2017) señalan que no hay diferencias entre el hombre y la mujer, ambos pueden aportar lo mismo a la organización, sin embargo esto se puede ver afectado negativamente por el rol que deben cumplir en el hogar como es el cuidado de los hijos, quien tenga más compromisos en el hogar mermará su compromiso con la organización y por ende su productividad.

Por último, la variable edad estadísticamente demostró no ser significativa para la productividad, los estudios que se han realizado son contradictorios, por lo que la edad es una variable que no debe aislarse sino que debe considerar varios elementos, por ejemplo se espera que las 
RELACIÓN DEL FACTOR INDIVIDUAL CON LA FUNCIÓN INVESTIGACIÓN EN LAS UNIVERSIDADES PÚBLICAS.

personas de mayor edad dejen de producir porque están pensando en su próxima jubilación, sin embargo la experiencia que tienen y el compromiso con la organización es mayor que la de los jóvenes lo que influye en la mayoría de los casos para que sigan aportando, por el contrario en los jóvenes el compromiso con la organización es menor, pero pueden haber jóvenes que deseen hacer carrera en ella y ver una oportunidad de lograr crecimiento profesional, haciendo que sean más productivos (Salas y Alegre, 2017).

\section{REFERENCIAS BIBLIOGRÁFICAS}

Abdullah, S. y Elgaraihy, W. (2013). The Impact of Human Resource Management Practices, Organisational Culture, Organisational Innovation and Knowledge Management on Organisational Performance in Large Saudi Organisations: Structural Equation Modeling With Conceptual Framework. International Journal of Business and Management, 8(22), 120. DOI 10.5539/ijbm.v8n22p1.

Ahani, M., Bahrami, H. y Rostami, M. (2012). Determining and ranking dimensions of knowledge management implementation using Hicks model and fuzzy TOPSIS Technique. Management Science Letters, 721-730. Recuperado de http://www.econbiz.de/Record/determining-and-ranking-dimensions-of-knowledgemanagement-implementation-using-hicks-model-and-fuzzy-topsis-technique-ahanimona/10009720861.

Almanza, R., Calderón, P., Vargas, J., Casas, R. y Palomares, F. (2016). Aprendizaje y Desempeño Organizacional Bajo el Enfoque de las Teorías Organizacionales. Revista de Economía y Administración, 13(1), 83-94. Recuperado de https://www.uao.edu.co/sites/default/files/Revista\%20131\%20Julio\%2013\%20Arti\%CC\%81culo\%205.pdf.

Alshehhi, A. y Jasimuddin, S. (2016). A Framework for Organizational Knowledge Systems. International Journal of Business and Administrative Studies, 2(6), 194-201. Recuperado de 
RELACIÓN DEL FACTOR INDIVIDUAL CON LA FUNCIÓN INVESTIGACIÓN EN LAS UNIVERSIDADES PÚBLICAS.

https://www.researchgate.net/publication/324227188_A_FRAMEWORK_FOR_ORGANIZA TIONAL_KNOWLEDGE_SYSTEMS.

Amador, N., Aguirre, M., Anguiano, N. y Guízar Mendoza, J. (2018). Desempeño laboral de acuerdo al estado de salud del trabajador y el uso del móvil en organizaciones laborales. Nova Scientia, 10 (2), 423-440. Recuperado de file:///C:/Users/201500274UJAT/Desktop/jefatura\%20de\%20investigaci\%C3\%B3n/INFOR ME\%20RECTORIA/informe\%20rector\%202019/1406-6606-1-PB.pdf.

Apórtela, I. y Ponjuán, G. (2008). La Segunda Generación de la Gestión del Conocimiento: Un nuevo enfoque de la gestión del conocimiento. Ciencias de la Información, 39(1), 19-30. Recuperado de http://search.ebscohost.com/login.aspx?direct=true\&db=zbh\&AN=32455551\&lang=es\&site =ehost-live.

Argyris, C. (1991). The Use of Knowledge as a Test for Theory: The Case of Public Administration. Journal of Public Administration Research and Theory, 1(3), 337-354.

Awadh, A. (2007). Why Academics Fail to Utilize Academic-Funded Research Opportunities? An Empirical Study. International Journal Of Management, 24(4), 712-726. Recuperado de http://search.ebscohost.com/login.aspx?direct=true\&db=bth\&AN=31708166\&lang=es\&site =ehost-live.

Bandura, A. (1977). SELF-EFFICACY: TOWARD A UNIFYING THEORY OF BEHAVIORAL CHANGE. Psychologica Review, 84, 191-215.

Bavarsad, B., Rahimi, F., Beiranvand, T. y Jafarnea, S. (2016). Investigating the Relationship of Social Capital with Knowledge Management and Organizational Learning in Manufacturing Firms of Khuzistan Province. Entrepreneurship and Innovation Management Journal, 4(1), 34-47. Recuperado de http://oaji.net/articles/2016/353-1459562559.pdf.

Bland, C., Center, B., Finstad, D., Risbey, K. y Staples, J. (2006). The Impact of Appointment Type on the Productivity and Commitment of Full-Time Faculty in Research and Doctoral Institutions. Higher Education, 77(1), 89-123. Recuperado de 
RELACIÓN DEL FACTOR INDIVIDUAL CON LA FUNCIÓN INVESTIGACIÓN EN LAS UNIVERSIDADES PÚBLICAS.

http://search.ebscohost.com/login.aspx?direct=true \&db=a9h\&AN=19413799\&lang=es\&site =ehost-live.

Bontis, N. (1998). Intellectual capital: an exploratory study that develops measures and models. Management Decision, 36(2), 63-76. doi:10.1108/00251749810204142.

Bontis, N., Crossan, M. y Hulland, J. (2002). Managing An Organizational Learning System By Aligning Stocks and Flows. Journal of Management Studies 39(4), 437-469. DOI 10.1111/1467-6486.t01-1-00299 .

Broadbent, M. (1998). The phenomemnon of knowledge management: what does it mean to the information profession? Information Outlook, 23-36. Recuperado de http://citeseerx.ist.psu.edu/viewdoc/download?doi=10.1.1.473.463\&rep=rep1\&type=pdf.

Cárdenas, S., Cabrero, E. y Arellano, D. (2012). La difícil vinculación universidad-empresa en México. D.F., México: CIDE.

Chiavenato, I. (2009). Comportamiento Organizacional. La dinámica del éxito en las organizaciones. México: McGraw-Hill.

Consejo Nacional de Ciencia y Tecnología [CONACYT]. (2008). Programa Institucional 20082012. México, D.F. Recuperado de http://www.siicyt.gob.mx/siicyt/docs/contenido/Proglnst0812.pdf.

Consejo Nacional de Ciencia y Tecnología [CONACYT]. (2017). Padrón de beneficiarios. Obtenido de Menú del Sistema Nacional de Investigadores: http://www.conacyt.gob.mx/index.php/el-conacyt/sistema-nacional-deinvestigadores/archivo-historico

Consejo Nacional de Ciencia y Tecnología [CONACYT]. (2018). Padrón de beneficiarios. Obtenido de Menú del Sistema Nacional de Investigadores: https://www.conacyt.gob.mx/index.php/el-conacyt/sistema-nacional-de-investigadores 
RELACIÓN DEL FACTOR INDIVIDUAL CON LA FUNCIÓN INVESTIGACIÓN EN LAS UNIVERSIDADES PÚBLICAS.

Cortes, J. y Pérez, J. (2008). El aprendizaje organizacional: Reflexión desde la investigación aplicada en el grupo de estudios empresariales. Cuadernos de Administración, 39, 29-36. Recuperado de https://www.redalyc.org/pdf/2250/225020360003.pdf.

Davenport, T., De Long, D. y Beers, M. (1998). Successful knowledge management projects. Sloan Management Review, 39(2), 45-57. Recuperado de https://www.ischool.utexas.edu/ i385q-dt/readings/Davenport_DeLong-1998Successful.pdf.

Del Castillo, C. y Vargas, B. (2009). El proceso de gestión y el desempeño organizacional. Cuadernos de Difusión, 14(26), 57-80. Recuperado de http://search.ebscohost.com/login.aspx?direct=true\&db=bth\&AN=43901219\&lang=es\&site =ehost-live.

Drucker, P. (2000). La productividad del trabajador del conocimiento: máximo desafío. Harvard Deusto Business Review, (98), 4-16. Recuperado de http://www3.mapfre.com/estudios/boletin/N3/pdfs/productividad.PDF.

Elenkov, D. (2002). Effects of leadership on organizational performance in Russian companies. Journal of Business Research, (55), 467-480. Recuperado de http://www.sciencedirect.com/science/article/pii/S0148296300001740.

Griffis, S., Cooper, M., Golds, T. y Closs, D. (2004). Performance measurement: Measure selection based upon firm goals and information reporting needs. Journal of Business Logistics, 25(2), 95-118. Recuperado de http://onlinelibrary.wiley.com/doi/10.1002/j.21581592.2004.tb00183.x/pdf.

Guinot, J., Chiva, R. y Mallén, F. (2015). Altruismo y capacidad de aprendizaje organizativo: un estudio en las empresas mejor valoradas por los trabajadores en España. UNIVERSIA Business Review, 92-109. Recuperado de http://repositori.uji.es/xmlui/bitstream/handle/10234/153475/chiva\%202016.pdf?sequence= 1\&isAllowed=y.

Hadjinicola, G. y Soteriou, A. (2006). Factors Affecting Research Productivity of Production And Operations Management Groups: An Empirical Study. Journal Of Applied Mathematics y 
RELACIÓN DEL FACTOR INDIVIDUAL CON LA FUNCIÓN INVESTIGACIÓN EN LAS UNIVERSIDADES PÚBLICAS.

Decision Sciences, (2), 1-16. Recuperado de http://search.ebscohost.com/login.aspx?direct=true\&db=bth\&AN=23128366\&lang=es\&site =ehost-live.

Hall, R. (1963). The concept of bureaucracy: an empirical assessment. The American Journal of Sociology, 69(1), 32-40.

Hernández, D., Fernández, D. y Baptista, D. (2010). Metodología de la Investigación. México: Mc Graw Hill.

Huber, G. (1991). Organizational learning: the contributing processes and the literatures. Organization Science, 2(1), 88-115. Recuperado de http://www.researchgate.net/profile/George_Huber2/publication/215439842_Organizationa I_Learning_The_Contributing_Processes_and_the_Literatures/links/549aefa20cf2b803713 7169a.pdf.

Jyoti, J., Gupta, P. y Kotwal, S. (2011). Impact of Knowledge Management Practices on Innovative Capacity: A Study of Telecommunication Sector. Vision, 15(4), 315-330. DOI 10.1177/097226291101500402.

Khan, S., Rajasekar, J. y Al-Asfour, A. (2015). Organizational Career Development Practices: Learning from an Omani Company. International Journal of Business and Management; 10(9), 88-98.

Kim, D. (1993). El vínculo entre el individuo y el aprendizaje organizacional. Sloan Management Review, 35(1), 37-50. Recuperado de http://sloanreview.mit.edu/article/the-link-betweenindividual-and-organizational-learning/.

Lee, H. y Choi, B. (2003). Knowledge Management Enablers, Processes, And Organizational Performance: An Integrative View And Empirical Examination. Journal Of Management Information Systems, 20(1), 179-228. Recuperado de http://search.ebscohost.com/login.aspx?direct=true\&db=bth\&AN=10221618\&lang=es\&site =ehost-live. 
RELACIÓN DEL FACTOR INDIVIDUAL CON LA FUNCIÓN INVESTIGACIÓN EN LAS UNIVERSIDADES PÚBLICAS.

Lin, H. (2007). Knowledge sharing and firm innovation capability: An empirical study. International Journal of Manpower, 28(34), 315-332. Recuperado de http://mapule276883.pbworks.com/f/Knowledgs+sharing+and+firm+inniovation.pdf.

Lin, H. y Lee, G. (2006). Effects of socio-technical factors on organizational intention to encourage knowledge sharing. Management Decision, 44(1), 74-88. Recuperado de http://www.emeraldinsight.com/journals.htm?issn=0025-

$1747 \&$ volume $=44 \&$ issue $=1 \&$ articleid $=1537858 \&$ show $=$ html.

López, Á., Pérez, A. y Valle, R. (2009). Knowledge As A Mediator Between HRM Practices And Innovative Activity. Human Resource Management, 48(4), 485-503. Recuperado de http://search.ebscohost.com/login.aspx?direct=true\&db=bth\&AN=43494887\&lang=es\&site =ehost-live.

Mollick, E. (2012). People And Process, Suits And Innovators: The Role Of Individuals In Firm Performance. Strategic Management Journal, 33(9), 1001-1015. Recuperado de http://search.ebscohost.com/login.aspx?direct=true\&db=bth\&AN=77656382\&lang=es\&site =ehost-live.

Nonaka, I., Byosiere, P., Borucki, C. y Konnot, N. (1994). Organizational knowledge creation theory: A first comprehensive test. International Business Review, 3(4), 337-351, Recuperado de http://www.ai.wu.ac.at/ kaiser/birgit/Nonaka-PapersAlfred/IBR\%20Nonaka.pdf.

Ordónez, P. y Lytras, M. (2008). Competencies and human resource management: implications for organizational competitive advantage. Journal of Knowledge Management, 12(6), 4855. Recuperado de https://www.researchgate.net/publication/220363490_Competencies_and_human_resourc e_management_implications_for_organizational_competitive_advantage.

Organización de las Naciones Unidas para la Educación, la Ciencia y la Cultura [UNESCO]. (Octubre de 1998). La educación superior en el siglo XXI: Visión y acción. Conferencia Mundial sobre la Educación Superior (págs. 1-137). París: Recuperado de 
RELACIÓN DEL FACTOR INDIVIDUAL CON LA FUNCIÓN INVESTIGACIÓN EN LAS UNIVERSIDADES PÚBLICAS.

http://unesdoc.unesco.org/images/0011/001163/116345s.pdf. Obtenido de Conferencia Mundial sobre la Educación Superior.

Parra, C. y Calderón, G. (2013). Formación y desempeño: un análisis de caso en empresas manufactureras grandes. Pensamiento y Gestión, (34), 137-160. Recuperado de http://search.ebscohost.com/login.aspx?direct=true\&db=a9h\&AN=91100294\&lang=es\&site =ehost-live.

Pedraza, E., Amaya, G. y Conde, M. (2010). Desempeño laboral y estabilidad del personal administrativo contratado de la Facultad de Medicina de la Universidad del Zulia. Revista de Ciencias Sociales, 16(3), 493-505. Recuperado en http://www.scielo.org.ve/pdf/rcs/v16n3/art10.pdf.

Pérez, S., Montes, J. y Vázquez, C. (2006). Human Resources Management As A Determining Factor In Organisational Learning. Management Learning, 37(2), 215-239. Recuperado de http://www2.warwick.ac.uk/fac/soc/wbs/conf/olkc/archive/oklc5/papers/g-1_perez.pdf.

Pérez, A., Rodríguez, V. y Sotelo, F. (2012). Altruismo vs. egoísmo en la educación humanista. CIFE, 14(21), 197-214.

Porter, M. y Wayland, R. (1992). Capital Disadvantage: America's Failing Capital Investment System. Harvard Business Review. 70(5), 65-82. Recuperado de http://search.ebscohost.com/login.aspx?direct=true\&db=bth\&AN=9301105367\&lang=es\&si te=ehost-live.

Ruiz, J., Silva, N. y Vanga, M. (2008). Ética empresarial y el desempeño laboral en Organizaciones de Alta Tecnología (OAT). Revista Venezolana de Gerencia, 13(43), 417441. Recuperado de http://www.scielo.org.ve/pdf/rvg/v13n43/art06.pdf.

Saadat, V. y Saadat, Z. (2016). Organizational Learning as a Key Role of Organizational Success. Procedia - Social and Behavioral Sciences, 230, 219-225. Recuperado de http://www.sciencedirect.com/science/article/pii/S1877042816311296.

Salas, A. y Alegre, J. (2017). The role of gender, age, job level and job security on physician's engagement in Spanish public hospitals. Universia Business Review, 36-51. Recuperado 
RELACIÓN DEL FACTOR INDIVIDUAL CON LA FUNCIÓN INVESTIGACIÓN EN LAS UNIVERSIDADES PÚBLICAS.

de

http://search.ebscohost.com/login.aspx?direct=true \&db=bth\&AN=126862960\&lang=es\&sit e=ehost-live.

Seleim, A., Ashour, A. y Bontis, N. (2007). Human capital and organizational performance: a study of Egyptian software companies. Management Decision, 45(4), 789-801. DOI 10.1108/00251740710746033.

Silva, A. y Flores, M. (2015). La motivación y su relación con la productividad laboral del personal administrativo de la empresa Electric S.A. de C.V. en Tijuana Baja California. Global Conference On Business y Finance Proceedings, 10(1), 733-742. Recuperado de http://search.ebscohost.com/login.aspx?direct=true\&db=bth\&AN=100834470\&lang=es\&sit e=ehost-live.

Singh, J. y Chand, P. (2018). A Review Analysis of Job Satisfaction as an Antecedent of Organizational Citizenship Behaviour. International Journal of Research and Analytical Reviews, 5(3), 718-726.

Toledo, J. (2009). El aprendizaje organizacional y la competitividad en una pequeña empresa: estudio de caso. Mercados y Negocios, 20, 5-25. Recuperado de file://C:/Users/201500274UJAT/Desktop/edo\%202018/jefe\%20depto/5113-16208-1SM.pdf.

Universidad Juárez Autónoma de Tabasco, [UJAT]. (2018). 2do Informe de Actividades 2017 Dr. José Manuel Piña Gutiérrez Rector. Villahermosa, Tabasco: Colección Justo Sierra.

Vásquez, F. (2010). Modelo de gestión del conocimiento para medir la capacidad productiva en grupos de investigación. Ciencia, docencia y tecnología, (41), 101-125. Recuperado de http://www.scielo.org.ar/scielo.php?pid=S1851-17162010000200005\&script=sci_arttext.

Wilson, J. y Musick, M. (1997). Work and Volunteering:The Long Arm of the Job. Social Forces, 76(1), 251-272. 
Original

RELACIÓN DEL FACTOR INDIVIDUAL CON LA FUNCIÓN INVESTIGACIÓN EN LAS UNIVERSIDADES PÚBLICAS.

Yanez, B., Hernandez, F. y Moreno, J. (2017). From Training to Organizational Behavior: A Mediation Model through Absorptive and Innovative Capacities. Frontiers in Psychology, 8, $1-22$.

Zack, M., McKeen , J. y Singh, S. (2009). Knowledge Management And Organizational Performance: An Exploratory Analysis. Journal of knowledge management, 13(6), 392409. Recuperado de http://www.emeraldinsight.com/journals.htm?articleid=1817427.

Zakaria, N. y Taiwo, A. (2013). The effect of team leader skills and competencies team: A structural equation modelling approach. Asian Ciencias Sociales, 9(7), 151:161. Recuperado de http://www.ccsenet.org/journal/index.php/ass/article/view/27940. 